\title{
Orientation and Structure of Palladium Particles Formed by Evaporation on Alkalihalide Crystals
}

\author{
By Koji Fukaya*, Shozo Ino** and Shiro Ogawa***
}

\begin{abstract}
The orientation and structure of palladium particles evaporated onto $\mathrm{NaCl}, \mathrm{KCl}, \mathrm{KBr}$ and $\mathrm{KI}$ cleaved in ultrahigh vacuum have been studied by electron diffraction and electron microscopy. It is concluded that the external shape of the epitaxial particles with the (001) orientation, showing a square shape, is generally an octahedron, although they have so far been considered to be a square pyramidal shape. The particles with the octahedral form grow at the nucleation stage, and then their shape changes gradually into a form truncated by six $\{100\}$ faces in the subsequent stage. This shape is similar to Wulff polyhedron. The particles with the (001), (111) and (110) orientations have been observed. Furthermore, three kinds of the multiply-twinned particles of palladium have actually been observed on electron micrographs. Their particle sizes are generally very large as compared with those predicted by Ino.
\end{abstract}

(Received May 17, 1978)

\section{Introduction}

A number of detailed experimental researches on the epitaxial growth ${ }^{(1)(2)}$ of thin metal films have so far been made by using electron diffraction and electron microscopy. One of the most interesting results is discovery of the multiply-twinned particles. Allpress and Sanders $^{(3)}$ and Mihama and Yasuda ${ }^{(4)}$ observed abnormal (111) spots in electron diffraction patterns taken from thin gold films. Ino $^{(5)}$ and Ino and Ogawa ${ }^{(6)}$ studied in detail the origin of the abnormal (111) spots by using the technique of dark field electron microscopy, and the complex particle structures were elucidated. They found that the particles are composed of tetrahedra in a twin relation with each other, and the particles were called "multiply twinned particles". The structures were confirmed by later investigations using evaporated films ${ }^{(7)(8)(9)}$ and fine metal

* The Research Institute for Iron, Steel and Other Metals, Tohoku University, Sendai. Present address: Nishiohigashi High School, 1-4 Daigo, Ojima-cho, Nishio-shi, Aichi-ken 445, Japan.

** The Research Institute for Iron, Steel and Other Metals, Tohoku University, Sendai 980, Japan.

*** The Research Institute for Iron, Steel and Other Metals, Tohoku University, Sendai. Present address: Shibaura Institute of Technology, 3-9-14 Shibaura Minato-ku, Tokyo 108, Japan.

Trans. JIM particles $^{(10)}$ formed by vaporization in argon gas. These studies were mainly done on gold and silver films. However, there were a few papers which described on metals except gold and silver. In the case of palladium, an investigation $^{(11)}$ on thin films evaporated onto $\mathrm{NaCl}$ and $\mathrm{KCl}$ was presented, in which the structure was analysed only by electron diffraction patterns. The existence of the multiplytwinned particles were concluded only by diffraction spots.

The present experiment has started to study the growth mechanism of evaporated palladium films in relation to the multiply-twinned particles. The orientation and structure of palladium fine particles formed by evaporation onto the clean surfaces of $\mathrm{NaCl}, \mathrm{KCl}, \mathrm{KBr}$ and $\mathrm{KI}$ cleaved in ultrahigh vacuum have been investigated by analysis of electron diffraction patterns and electron micrographs. The latter were taken not only with normal incidence but also with inclined incidence of $45^{\circ}$ to analyse external shapes of the epitaxial particles showing a square shape with the (001) orientation.

Orientations of the Wulff polyhedral particles observed in the present work are mainly (001) but sometimes (111) and (110). Size distribution of the (001) epitaxial particles has been investigated to know the growth mechanism of the Wulff polyhedral particles.

1978 Vol. 19 
It is very rare that these particles are found in evaporated films, although they have been observed in the case of fine metal particles formed by vaporization in argon $\operatorname{gas}^{(12)(13)}$.

Three kinds of multiply twinned particles of palladium proposed by $\mathrm{Ino}^{(5)}$ and Ino and Ogawa $^{(6)}$ have actually been observed on electron micrographs. Their particle sizes are generally very large as compared with those predicted by Ino ${ }^{(14)(15)}$.

\section{Experimental Procedure}

Alkalihalide crystals $\mathrm{NaCl}, \mathrm{KCl}, \mathrm{KBr}$ and $\mathrm{KI}$ were used as substrates and they were cleaved in ultrahigh vacuum. There were some critical temperatures above which smooth surfaces could not be obtained by cleavage. These temperatures were about $150^{\circ} \mathrm{C}$ for $\mathrm{NaCl}, \mathrm{KCl}$ and $\mathrm{KBr}$ and about $120^{\circ} \mathrm{C}$ for KI. The residual gas pressure was 6-13 $\times$ $10^{-8} \mathrm{~Pa}\left(=5-10 \times 10^{-10} \mathrm{mmHg}\right)$ before evaporation and less than $3-10 \times 10^{-6} \mathrm{~Pa}$ during the evaporation. Palladium was evaporated from an electrically heated tungsten filament in helical shape. The deposition rate was about $5-10 \mathrm{~nm} / \mathrm{min}$. The mean thickness of the films ranged from 1 to $50 \mathrm{~nm}$.

The substrates were cooled down to room temperature after deposition and then carbon was condensed onto the thin films. The palladium films were isolated from the substrates in water and examined by a transmission electron microscope. To investigate the external shape of palladium particles, the samples were occasionally inclined by $45^{\circ}$ by using a special sample holder which was made by cutting obliquly a conventional one.

\section{Experimental Results}

\section{Palladium on $\mathrm{NaCl}, \mathrm{KCl}$ and $\mathrm{KBr}$}

(1) External shape of epitaxial particles with the (001) orientation

Photograph 1 shows an electron diffraction pattern (a) and an electron micrograph (b) taken from a palladium film $15 \mathrm{~nm}$ thick formed on $\mathrm{NaCl}$ at $300^{\circ} \mathrm{C}$. Many squareshaped particles have the (001) orientation;

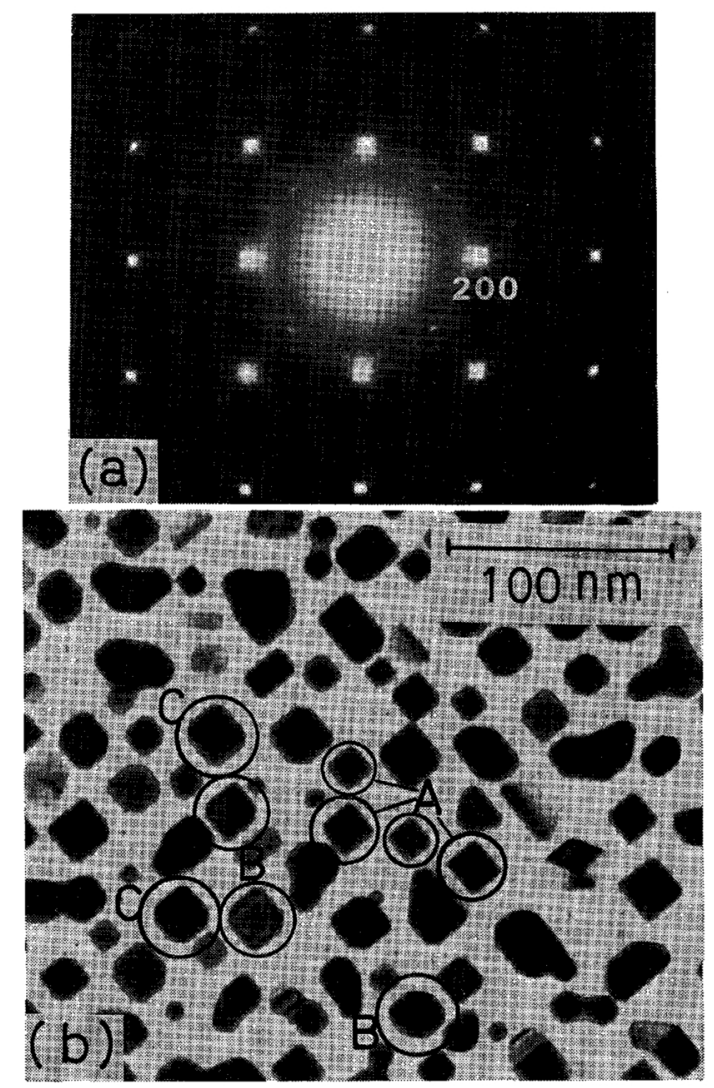

Photo. 1 Electron diffraction pattern (a) and electron micrograph (b) taken from a palladium film $15 \mathrm{~nm}$ thick evaporated onto $\mathrm{NaCl}$ at $300^{\circ} \mathrm{C}$.

$(001)_{\mathrm{Pd}} / /(001)_{\mathrm{NaCl}}$ and $\langle 110\rangle_{\mathrm{Pd}} /\langle 110\rangle_{\mathrm{NaCl}}$. Their external shape has so far been supposed to be a square pyramidal form surrouded by four $\{111\}$ faces and a (001) plane, the latter adhering to the substrate as illustrated in Fig. 1(a).

In order to investigate the actual external shape of the (001) particles, electron micrographs were taken from the films which were inclined by $45^{\circ}$ about the axis parallel to the [100] direction of $\mathrm{NaCl}$. By this rotation the (011) plane becomes perpendicular to the electron beam, as seen in the diffraction pattern of Photo. 2(a). Photograph 2(b) is a bright field image, and (c) is a dark field image which was taken by using one of the (111) Bragg reflections. The external shape can be determined from the profile and equi-thickness fringes appearing in (b) and (c). 


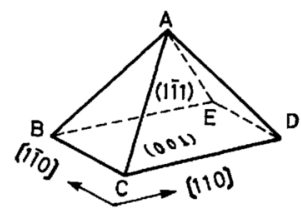

(a)

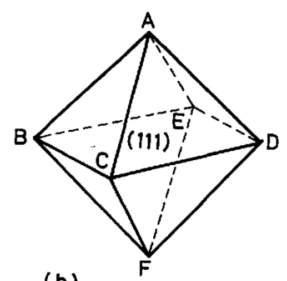

(b)

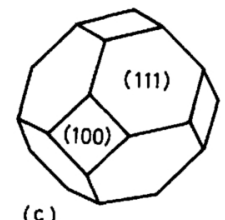

(c)

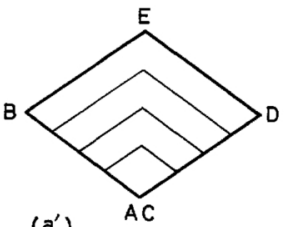

$\left(a^{\prime}\right)$

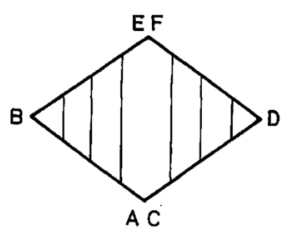

$\left(b^{\prime}\right)$

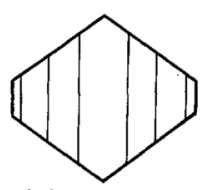

$\left(c^{\prime}\right)$

s!

Fig. 1 Illustration of shapes and equi-thickness fringes in the (001) epitaxial particles. (a) and ( $\left.a^{\prime}\right)$, (b) and ( $\left.b^{\prime}\right)$, and (c) and $\left(c^{\prime}\right)$ correspond to a half of an octahedron, a full octahedron and a truncated octahedron, respectively.

Figure 1 illustrates relations between the external shapes and their equi-thickness fringes which can be expected when the particles are inclined by $45^{\circ}$. Figure 1(a) corresponds to a half of an octahedron shown in (b), and its (001) plane is paralled to the substrate. When the particle (a) is rotated by $45^{\circ}$ about $[100]_{\mathrm{Pd}}$ axis $\overline{\mathrm{BD}}$, the electron beam becomes parallel to $\overline{\mathrm{AC}}$ direction, and a rhombic shape with equi-thichness fringes parallel to edges $\overline{\mathrm{EB}}$ and $\overline{\mathrm{ED}}$ as illustrated in $\left(\mathrm{a}^{\prime}\right)$ is expected. On the other hand, the octahedral particle shown in (b) produces equi-thickness fringes parallel to $\overline{E A}$ as illustrated in $\left(b^{\prime}\right)$, although the external shape becomes rhombic very similar to $\left(a^{\prime}\right)$. In the dark field image of Photo. 2(c), a few particles (in the rectangular frame) show actually the equi-thickness fringes corresponding to Fig. 1( $\left.\mathrm{a}^{\prime}\right)$, but most of the particles (in the circular frames) show the fringes as in $\left(b^{\prime}\right)$ to be expected from the octahedron (b).

Similar results were obtained in the case of $\mathrm{KCl}$ and $\mathrm{KBr}$ substrates. Photograph 3

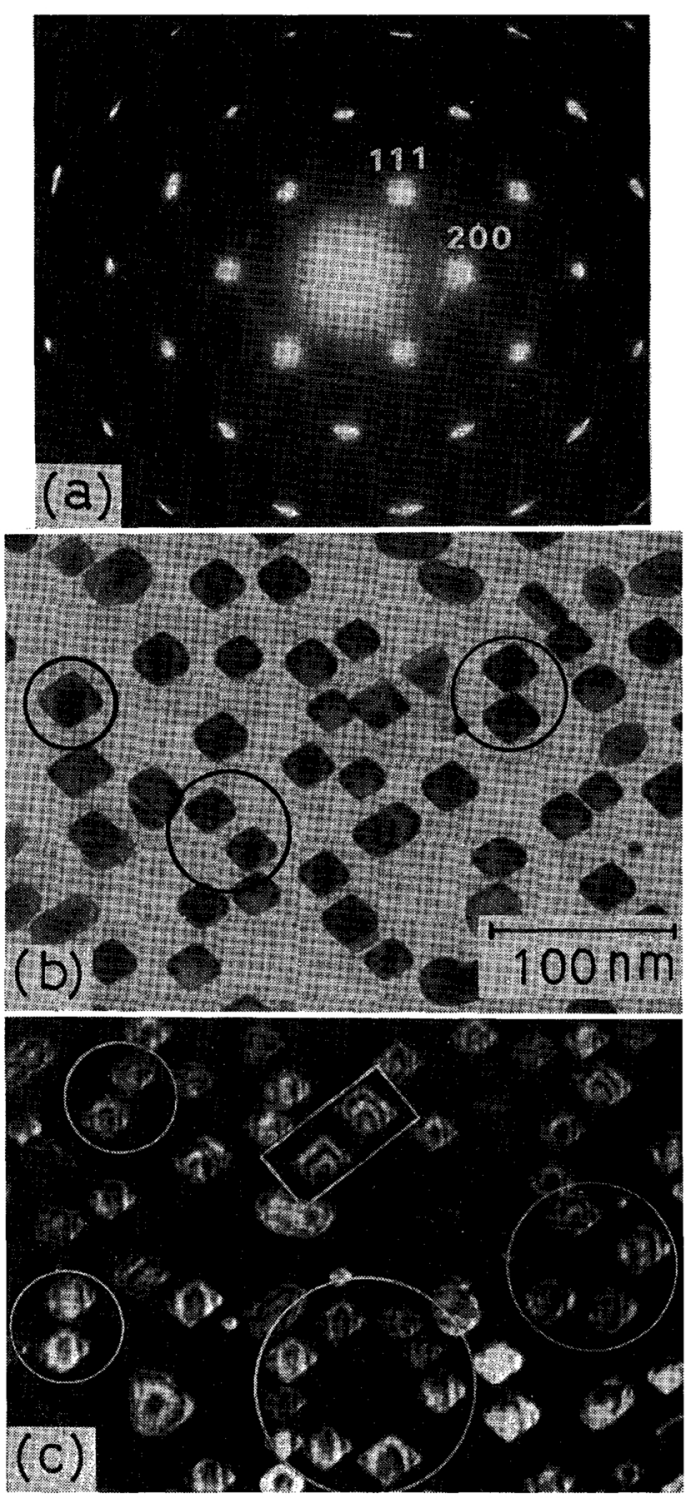

Photo. 2 Electron diffraction pattern and bright field and dark field images taken from the same sample as that in Photo. 1 which was inclined by $45^{\circ}$ about the $[100]_{\mathrm{NaCl}}$ axis.

shows such an example taken from a palladium film $10 \mathrm{~nm}$ thick formed on $\mathrm{KBr}$ at $305^{\circ} \mathrm{C}$. This electron micrograph was taken by the same condition as in Photo. 2(b). Most of the epitaxial particles also show the equi-thickness fringes to be explained by the octahedral shape shown in Fig. 1(b).

In the case of $\mathrm{KCl}$, the general features are 


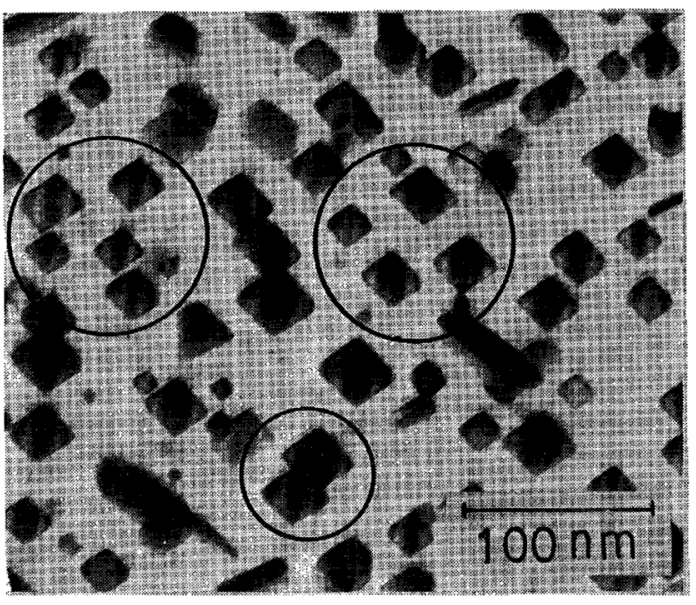

Photo. 3 Electron micrograph taken from a palladium film $10 \mathrm{~nm}$ thick evaporated onto $\mathrm{KBr}$ at $305^{\circ} \mathrm{C}$, the sample being inclined by $45^{\circ}$ about $[100]_{\mathrm{KBr}}$ axis.

very similar to the cases of $\mathrm{NaCl}$ and $\mathrm{KBr}$. Thus, it is concluded that most of the epitaxial particles of palladium with the (001) orientation evaporated on $\mathrm{NaCl}, \mathrm{KCl}$ and $\mathrm{KBr}$ have the octahedral shape in Fig. 1(b) and not the pyramidal shape in Fig. 1(a) which has for a long time been believed to be the form of the epitaxial particles with the (011) orientation.

\section{(2) Growth process of Wulff polyhedral particles}

The octahedral particles observed in Photo. 1 are occasionally not a perfect octahedron but truncated by $\{100\}$ planes. The external shape of the particles can be classified into three; octahedron (particle A), octahedron truncated by two $\{100\}$ faces (particle B) and octahedron truncated by six $\{100\}$ faces (particle C). The external shape of the particle $\mathrm{C}$ is illustrated in Fig. 1(c) and called cubooctahedron $^{(12)(13)}$. The geometrical shape is very similar to the Wulff polyhedron of $f c c$ structure which is a thermal equilibrium shape and has the minimum total surface energy among other shapes.

Figure 2 represents size distributions of the particles with the shapes $A, B$ and $C$ which were observed in an epitaxial palladium film grown on $\mathrm{NaCl}$ at $300^{\circ} \mathrm{C}$, (a) and (b) cor-

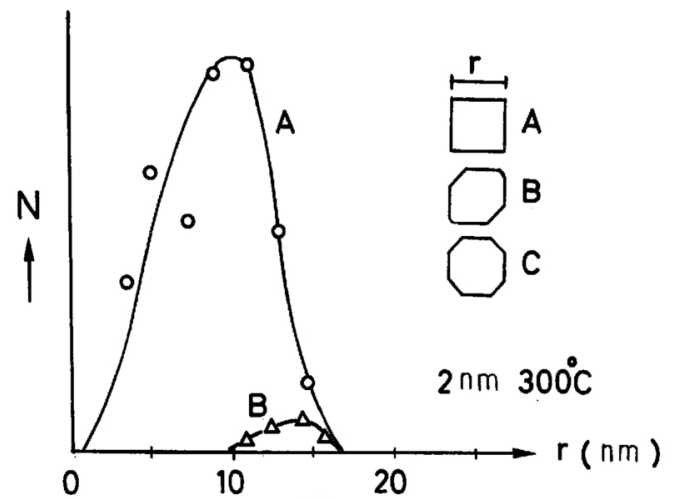

(a)

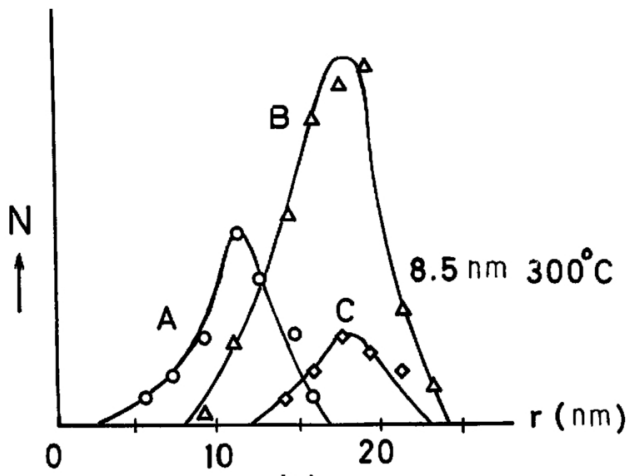

(b)

Fig. 2. Size distribution of epitaxial particles with the (001) orientation. In (a) and (b), the mean thicknesses are 2 and $8.5 \mathrm{~nm}$, respectively, the films being formed on $\mathrm{NaCl}$ at $300^{\circ} \mathrm{C}$.

responding to 2 and $8.5 \mathrm{~nm}$ in the mean thickness, respectively. The abscissa represents the diameter of particles in $\mathrm{nm}$ unit and the ordinate the number of particles $\mathrm{N}$ in an arbitraly unit. As seen in Fig. 2(a), most particles have the octahedral shape $A$ and a few have $B$ at the beginning stage of evaporation. The particle $\mathrm{C}$ can seldom be found. In the thicker film, however, the number of particle $\mathrm{A}$ is reduced, while that of particle B is increased and particle $\mathbf{C}$ appears, as seen in Fig. 2(b). The smaller particles show actually the fine octahedral shape $A$ and the larger ones the shape B or C. It is worthy of notice that the mean particle size of the octahedral particles $A$ is scarcely increased in spite of increase in the film thickness from (a) to (b). When the substrate temperature was elevated to $350^{\circ} \mathrm{C}$, similar phenomena were observed, 


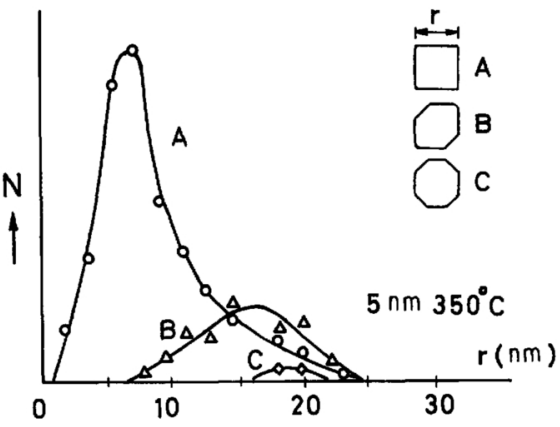

(a)

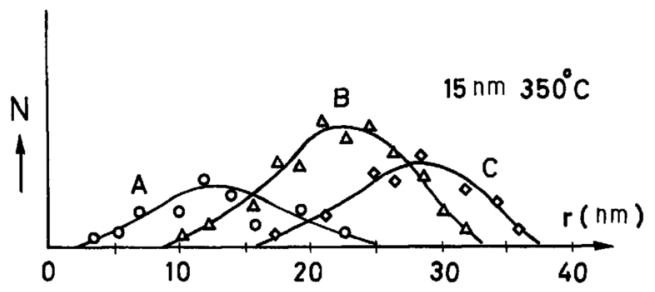

(b)

Fig. 3 Size distribution of epitaxial particles with the (001) orientation. In (a) and (b), the mean thicknesses are 5 and $15 \mathrm{~nm}$, respectively, the films being formed on $\mathrm{NaCl}$ at $350^{\circ} \mathrm{C}$.

as illustrated in Fig. 3(a) and (b), except that the particle sizes are somewhat larger and the particle densities are consequently smaller. On the basis of the experimental results, the growth process may be inferred in such a way that the octahedral particles A nucleate at the beginning stage of evaporation, then they change gradually their shape to a transient one, that is, to the particles $B$ in the next stage and are transformed into the particles $\mathrm{C}$ in the later stage.

The Wulff polyhedral particles have a tendency to grow when the substrate temperature is higher than $250^{\circ} \mathrm{C}$ and the vacuum is very high. When the degree of vacuum is not high, that is, the residual gas pressure is higher than about $1.3 \times 10^{-4} \mathrm{~Pa}$, the Wulff polyhedral particles could seldom be found. Contamination of the substrate must prevent the growth of the Wulff polyhedral particles; most particles grown on the clean substrates cleaved in vacuum were Wulff polyhedra, but they were rare on the substrates cleaved in air.

\section{Palladium on KI}

Palladium particles formed on KI show some different features with respect to the density, the shape and the orientation of particles, formation of multiply twinned particles etc., when compared with the cases on $\mathrm{NaCl}$, $\mathrm{KCl}$ and $\mathrm{KBr}$. Photograph 4(a) is an electron diffraction pattern and (b) and (c) electron micrographs taken from a palladium film $10 \mathrm{~nm}$ thick evaporated onto $\mathrm{KI}$ at $325^{\circ} \mathrm{C}$. The density of particles grown on $\mathrm{KI}$ is lower than that of particles on $\mathrm{NaCl}, \mathrm{KCl}$ and $\mathrm{KBr}$, and they have generally fine crystal habits, as seen in (b) and (c). Octahedral particles $\mathrm{A}$ and Wulff polyhedral ones $\mathrm{C}$ with the (001) orientation are seen. Particle $\mathbf{C}^{\prime}$ has a typical shape extensively truncated by six $\{100\}$ faces.

The diffraction pattern in Photo. 4(a) shows the existence of the (111) orientation, although it is very weak in the case of $\mathrm{NaCl}$ as seen in Photo. 1(a). A triangular particle $\mathrm{T}$ in Photo. 4(b) has the (111) orientation. Its external shape is considered not to be a tetrahedron shown in Fig. 4(a), but to be a tetrahedron extensively truncated by a (111) face parallel to the substrate, i.e. the particle is a thin plate, as illustrated in Fig. 4(b). It shows scarcely equi-thickness fringes, while a tetrahedron should do them. Particles $\mathbf{H}$ are octahedra with the (111) orientation in which one of the (111) faces is parallel to the substrate surface, as illustrated in Fig. 4(c). They are often truncated by six $\{100\}$ faces, as illustrated in Fig. 4(d), and their external shape is similar to Wulff polyhedron.

A rhombic particle $O$ in Photo. 4(b) has also an octahedral shape with the (110) orientation, differing from the multiply twinned particles shown in Fig. 5(b). The shape corresponds entirely to that observed from the sample inclined by $45^{\circ}$, as seen in Photo. 2(b), (c) and Photo. 3, and is explained by Fig. 1(b) and (b').

Another distinguished feature of palladium particles grown on $\mathrm{KI}$ is that they show a variety of the shape. Needle-like particles $\mathrm{N}$ and very long rectangular particles $\mathrm{Rc}$ are occasionally observed, as shown in Photo. 


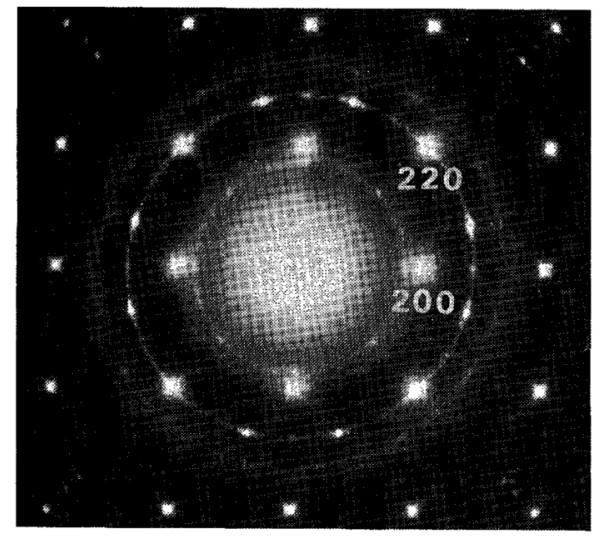

(a)

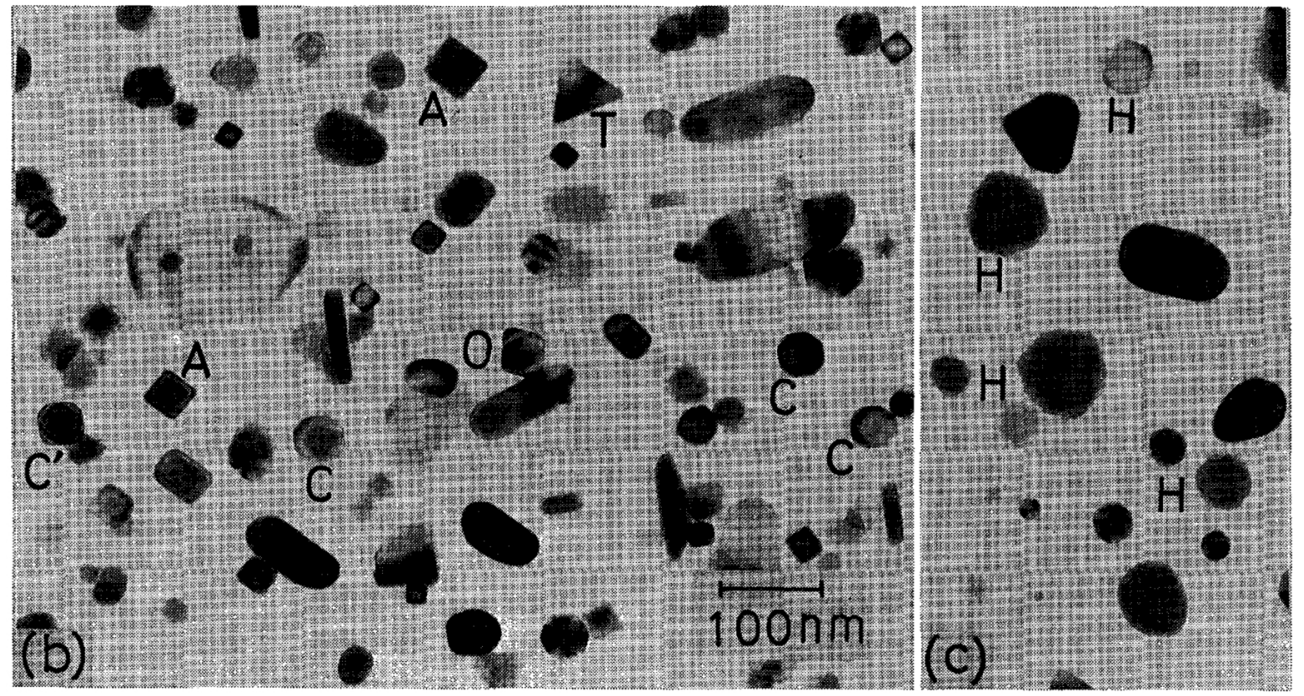

Photo. 4 Electron diffraction pattern and electron micrographs taken from a palladium film $10 \mathrm{~nm}$ thick evaporated onto $\mathrm{KI}$ at $350^{\circ} \mathrm{C}$.

5(a). They should be classified into a kind of whisker. The needle direction coincides with one of the $\langle 100\rangle$ directions of KI. Very thin and large particles with the (111) orientation are also observed, as seen in Photo. 5(b).

\section{Multiply twinned particles}

Ino $^{(5)}$ and Ino and Ogawa ${ }^{(6)}$ observed three kinds of the multiply twinned particles on electron micrographs taken from evaporated gold particles. In the case of palladium studied by Ogawa et al. ${ }^{(11)}$ the existence of the multiply twinned particles was concluded only by analyzing diffraction patterns taken from thin evaporated films.
Three kinds of the multiply twinned particles similar to the case of gold were confirmed by electron micrographs in the present work. Photograph 6(a), (b) and (c) were taken from palladium particles formed on KI. Particles $\mathrm{P}, \mathbf{R}$ and I correspond to pentagonal, rhombic and hexagonal particles as illustrated in Fig. $5(a)$, (b) and (c) ${ }^{(5)(6)}$, respectively. The tetrahedral nucleus of a pentagonal particle takes generally the (111) orientation as seen in Fig. 5(a), and the equi-thickness fringes as illustrated in Fig. 6(a) should appear. A pentagonal particle in Photo. 6(b) just corresponds to this orientation. However, a pentagonal particle $\mathbf{P}$ in Photo. 6(a) has a 
(a)

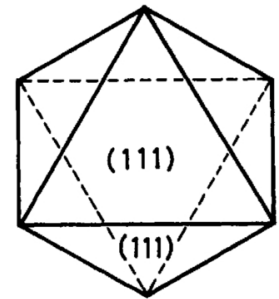

(c)

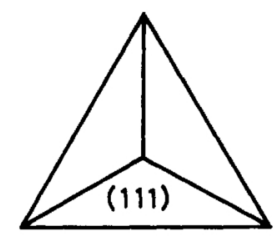

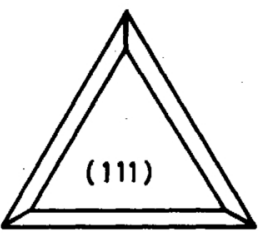

(b)

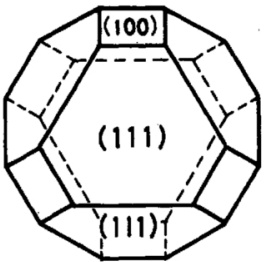

(d)
Fig. 4 Illustration of shapes of particles with the (111) orientation. (a), (b), (c) and (d) are a tetrahedron, a truncated tetrahedron, an octahedron and a truncated octahedron, respectively.

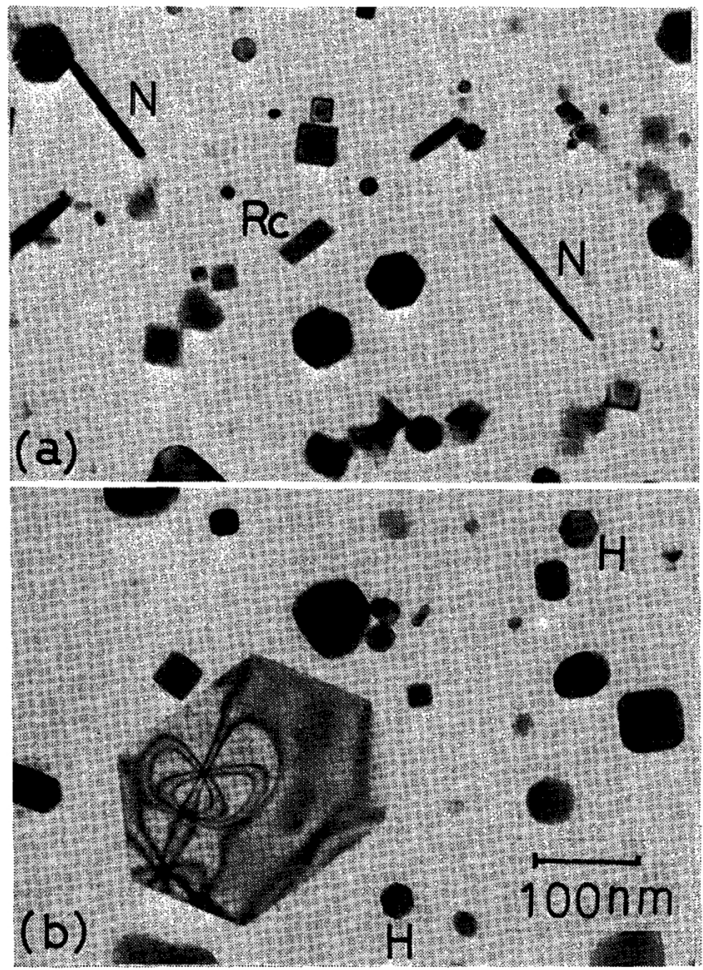

Photo. 5 Electron micrographs taken from a palladium film $10 \mathrm{~nm}$ thick evaporated onto $\mathrm{KI}$ at $350^{\circ} \mathrm{C}$.

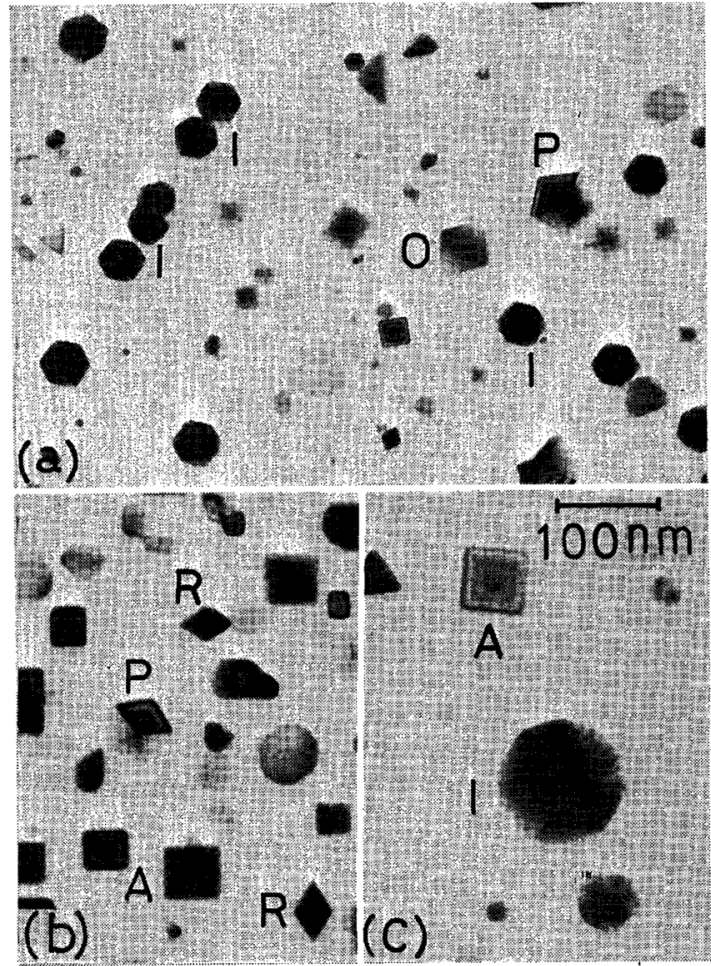

Photo. 6 Electron micrographs showing the multiply twinned particles, taken from a palladium film $10 \mathrm{~nm}$ thick evaporated onto KI at $300^{\circ} \mathrm{C}$. Pentagonal and hexagonal particles are seen in (a), and rhombic particles in (b). The largest hexagonal particle observed in the present experiment is seen in (c). It shows some deformed equi-thickness fringes.

five-fold symmetry about the incident electron beam direction, corresponding to a view from $\overline{\mathrm{OA}}$ direction in Fig. 5(a). In this case, the equi-thickness fringes should appear as illustrated in Fig. $6(\mathrm{~b})$, and such fringes are actually seen in Photo. 6(a). The plane perpendicular to $\overline{\mathrm{OA}}$ direction is (110), and so the nucleus of the pentagonal particle in Photo. 6(a) takes the (110) orientation.

Sizes of the multiply twinned particles found in thin palladium films formed on KI are generally very large as compared with the case of gold $^{(5)(6)}$. A mean particle size of the icosahedral particles is about $40 \mathrm{~nm}$ in diameter. The largest diameter found in the case of palladium formed on $\mathrm{KI}$ is nearly $100 \mathrm{~nm}$, as seen in Photo. 6(c). This size is far larger than the critical size $26.39 \mathrm{~nm}$ 

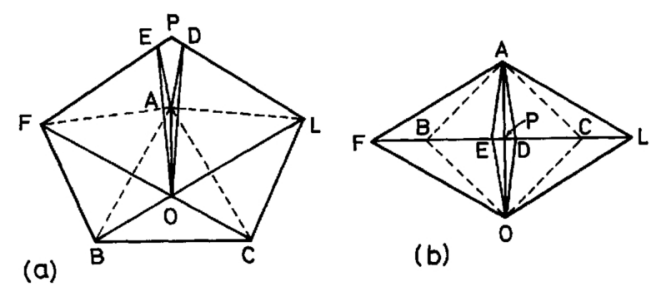

(b)

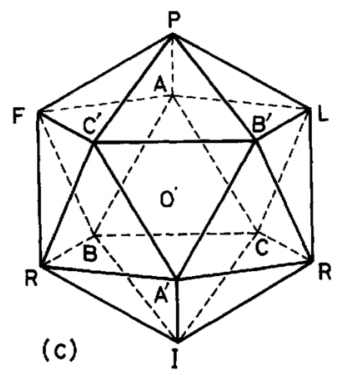

Fig. 5 Multiply twinned particles observed in palladium films. (a), (b) and (c) are a pentagonal, a rhombic and an icosahedral particle, respectively.

calculated by $\operatorname{Ino}^{(14)(15)}$.

\section{Discussion and Conclusion}

The epitaxial particles with the (001) orientation showing electron-microscopic images of a square shape have so far been considered to have a square pyramidal shape, a half of an octahedron. In the present observation electron micrographs were taken from palladium films inclined by $45^{\circ}$ from the normal incidence and it was revealed that most of the squareshaped particles had really an octahedral shape, although a few pyramidal shapes were actually observed. Octahedral particles A with the (001) orientation nucleate at the beginning stage of evaporation, then change their form gradually to those truncated by $\{100\}$ planes, resulting in the Wulff polyhedral shape at the later stage.

According to the electron-microscopic observation, the Wulff polyhedral particles with the (001), (111) and (110) orientations were observed and they show a truncated square, a truncated hexagonal and a truncated rhombic shape, respectively. The Wulff polyhedral shape has so far scarcely been observed in the case of evaporated films. However, particles with this shape grow easily in palladium films,

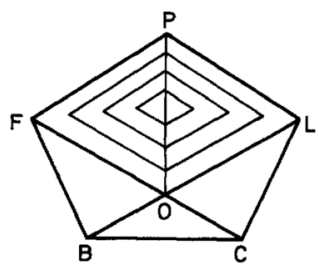

(a)

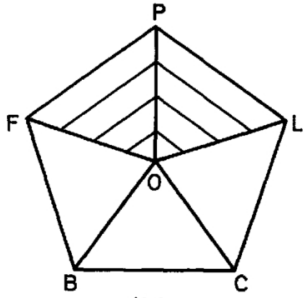

(b)
Fig. 6 Equi-thickness fringes observed in pentagonal particles. The nuclei in (a) and (b) have the (111) and (110) orientation, respectively.

if growth conditions such as the cleanness and temperature of substrates are controlled as in the present experiments. This shape has already been observed in fine metal particles formed by vaporization in argon $\operatorname{gas}^{(12)(13)}$.

Existence of the multiply twinned particles of palladium has been indicated by Ogawa et $a l^{(6)}$ only by analyzing diffraction patterns arising from palladium films. In the present experiment, three kinds of the multiply twinned particles have been confirmed to exist, directly from electron micrographs taken from palladium films evaporated on $\mathrm{NaCl}, \mathrm{KCl}, \mathrm{KBr}$ and $\mathrm{KI}$.

Sizes of the multiply-twinned particles in the present case are generally very large as compared with the case of gold and silver. The largest diameter found in a palladium film formed on KI was about $100 \mathrm{~nm}$, which was far larger than the critical size $26.39 \mathrm{~nm}$ calculated by Ino(14)(15). This calculation was based on the assumption of a perfect multiplytwinned structure that contains no imperfections such as dislocations, stacking faults, etc. but only the essential straining. On the other hand, the multiply-twinned particles actually observed contain often dislocations ${ }^{(9)}$, stacking faults ${ }^{(13)}$, inhomogeneous strainings ${ }^{(16)}$ etc. The icosahedral particle shown in Photo. 6(c) must contain some imperfections because the equi-thickness fringes are deformed. By introduction of some imperfections, the strain energy $\mathrm{W}^{(15)}$ should be reduced. Thus, it is possible for such particles to grow larger than the value calculated by Ino.

\section{REFERENCES}

(1) D. W. Pashley: Advan. Phys., 5 (1956), 173. 
(2) D. W. Pashley: Advan. Phys., 14 (1965), 327.

(3) J. G. Allpress and J. V. Sanders: Phil. Mag., 10 (1964), 645.

(4) K. Mihama and Y. Yasuda: J. Phys. Soc. Japan, 21 (1966), 1166.

(5) S. Ino: J. Phys. Soc. Japan, 21 (1966), 346.

(6) S. Ino and S. Ogawa: J. Phys. Soc. Japan, 22 (1967), 1365.

(7) J. G. Allpress and J. V. Sanders: Surface Sci., 7 (1967), 1.

(8) E. Gillet and M. Gillet: J. Crystal Growth, 13/14 (1972), 212; ibid. Thin Solid Films, 15 (1973), 249.

(9) T. Komoda: Japan. J. Appl. Phys., 7 (1968), 27.
(10) K. Kimoto and I. Nishida: J. Phys. Soc. Japan, 22 (1967), 940.

(11) S. Ogawa, S. Ino, T. Kato and H. Ota: J. Phys. Soc. Japan, 21 (1966), 1963.

(12) T. Hayashi, T. Ohno, S. Yatsuya and R. Uyeda: Japan. J. Appl. Phys., 16 (1977), 705.

(13) K. Kimoto and I. Nishida: Japan. J. Appl. Phys., 16 (1977), 491.

(14) S. Ino: J. Phys. Soc. Japan, 26 (1969), 1559.

(15) S. Ino: J. Phys. Soc. Japan, 27 (1969), 941.

(16) K. Yagi, K. Takayanagi, K. Kobayashi and G. Honjo: J. Crystal Growth, 28 (1975), 117. 